Proceedings of the Pakistan Academy of Sciences:

A: Physical and Computational Sciences 58(3): 59-65 (2021)

Copyright $(C$ Pakistan Academy of Sciences

ISSN (Print): 2518-4245; ISSN (Online): 2518-4253

https://doi.org/10.53560/PPASA(58-3)745

\title{
Design, Simulation, and Implementation of Connected IoT Wearable Devices in Healthcare Applications
}

\author{
Karim Bagneid ${ }^{1}$, Youssef Sherif ${ }^{1}$, Maryam Soliman ${ }^{1}$, and Mohamed Hussein ${ }^{2 *}$ \\ ${ }^{1}$ Misr International University, Cairo, Egypt \\ ${ }^{2 *}$ Military Technical College, Cairo, Egypt
}

\begin{abstract}
Internet of Things (IoT) is one of the most versatile tools in modern technology and can be applied in many different domains. But one domain has started proving its need for more IoT-based systems and healthcare businesses. The main objective of this paper is to design, simulate, and implement a connected IoT-Health Care system to assist patients needing constant care and monitoring. This system is based on an integrated wearable device containing several sensors that detect and record the patient's health information. This data will then be saved on an online database that is easily monitored and controlled through a mobile application to keep the personnel responsible for the patient's care, constantly up to date with any recordings or alarms from the patient.
\end{abstract}

Keywords: IoT, Healthcare, Health monitoring, Wearable device, Chronic disease, Mobile application

\section{INTRODUCTION}

Every year, 17 million people die from cardiovascular diseases all over the world. $80 \%$ of premature heart disease and strokes are preventable [1]. Thankfully, today's technology has helped us keep an eye on our loved ones even if we cannot be physically there. Healthcare projects have always been an integral part of the development of societies. With our minds uninterrupted by health issues that used to plague our ancestors, we are now free to put our efforts to bettering our society and developing technology for our and our children's sake.

To help in this issue, we decided to focus on personalized healthcare services that many people need, especially those with chronic diseases or mobility problems that need a constant eye on them, be it at home or in more serious cases in the hospital. The idea behind this paper is to develop a healthcare-oriented wearable device that can constantly monitor a patient's vital signs, i.e. heart rate, blood pressure, temperature, etc. Moreover, for the convenience of the patient and the medical team responsible for him, a database containing all the health information will be created to store everything from the vitals to their medical history, and the names of the nurses/medical staff and attending physicians taking care of the patient.

This database will be automatically updated through the wearable device and accessed through a secure mobile application tailor-made for the medical team. And in accordance with each patient's need, we will tackle the design and implementation of internet of things (IoT) based system that can provide the needed monitoring for a bed ridden patient.

\subsection{Literature Review}

Since the birth of the term IoT, there have been increasingly more work and research regarding the subject. For our purposes, we will focus on IoT projects in the healthcare domain [1-3]. The integration of IoT devices in systems can be considered one of the most crucial steps in the healthcare business [4]. It presents an opportunity to take out human response delay, where a machine can possibly take actions in case of emergencies, such as administering a dose of allergy medicine or insulin that can be potentially lifesaving [5]. There are several pieces of work preceding our project

Received: : June 2021; Accepted: September 2021

*Corresponding Author: Mohamed Hussein <mhusse5@mtc.edu.eg> 
some of which are: The IoT based smart healthcare kit proposed by Gupta et al. [6], the purpose of this kit is to data information such as heart rate, blood pressure, and electro cardiogram (ECG) using specialized biomedical sensors. And can also send an alarm to the physician detailing the patient's current status and all relevant information. To control this kit, they used a 2nd generation Intel Galileo board, which is an Arduino certified single board computer, that will process all the raw data from the sensors then output them as readable and comprehensible information for the doctor. The system also has an option for uploading this information on a database containing all of the recordings and medical history. Instead of monitoring the data using a mobile application, they deployed a web portal that could access the database server containing the live feed of the patient's vitals.

Another example is the IoT Enabled Remote Health Care DataAcquisition and smart transmission system by Kiran et al. [7] this model is a decisionmaking engine design to help with power efficiency by sending data only when the system decides the collected information is important enough.

This offers an alternative to the less power efficient constant or live transmission of data. This system contains only one medical sensor reading ECG data. Thanks to the decision-making engine, and the energy efficiency, this model achieved much better performance than other systems using burst transmission.

In the paper "Adopting the Internet of Things Technologies in Health Care System" by Chiuchisan et al. [2], a healthcare system for at risk patients at intensive care units (ICU) is proposed. It monitors the patients' vital signs and will alert the medical team in case of any emergency or even in the case of the patient starting to move. In addition to monitoring the medical data. This system will also use sensors from other devices such as the $\mathrm{XBOX}$ KinectTM to monitor patients' movements and environments needing extremely close observation.

Aside from the specialized IoT based healthcare systems, there exist a new and rapidly growing field that seems to have a lot of people interested. We are talking about the rise of the fitness band smart wearables. Which have revolutionized the way people think about their health. Calculating your heart rate or oxygen levels is no longer a thing that we only remember to do when feeling sick or admitted into the hospital.

We can now have all the reading at our disposal on our wrist. Examples of these products include the Apple Watch and the Samsung Galaxy Watch. Unfortunately, even though they are considered smart bands and have direct connections with cell phones to access some of its features.

The IoT aspect does not extend to the health monitoring services these devices offer i.e., it cannot be accessed from any other device or be used by medical professionals, it also lacks the features to save the health data locally or remotely, which makes them inefficient regarding our intended goal [8].

\section{MATERIALS AND METHODS}

\subsection{Proposed System}

The desired system consists of three components. First, the medical team will use the mobile application to access or modify the patient's health data. The second is the wearable device's embedded programming which will collect and process the data from the biomedical sensors [9]. And the third is the link interface between the application and our device that allows us to receive information or to

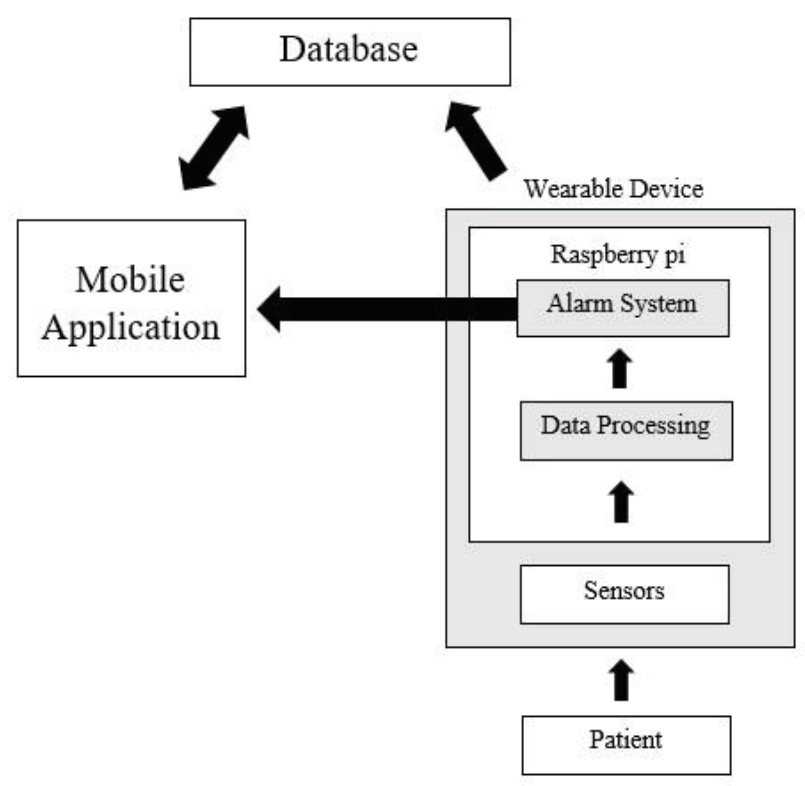

Fig. 1. proposed system architecture 
send commands to the wearable device.

Our system is comprised of a biomedical kit containing the sensors. we will use a Raspberry pi Zero setup to monitor the patient, which will receive the data from the sensors, process it, and then upload it to a database server to be saved and afterwards accessed any time through the mobile application.

As the wearable device aims for health monitoring especially for patients with chronic illnesses. The system will be recording some important vital signs via the biomedical sensors

\subsubsection{Body Temperature}

Body temperature ranges varies depending on the gender, age, time of the day, what part of the body the temperature is being measured from and the state of consciousness. Body temperature can be classified into the following:

- Hypothermia $<35^{\circ} \mathrm{C}$

- $36{ }^{\circ} \mathrm{C}<$ Normal $<37.2{ }^{\circ} \mathrm{C}$

- Fever $>38^{\circ} \mathrm{C}$

\subsubsection{Heart Rate}

Heart rate is number of times the heart beats per minute (bpm). Normal heart rate also varies according to age, gender, the body's physical needs and state of consciousness, as shown in Table 1 and Table 2 below.

Depending on diet, drugs, stress, hormonal status, environment, and illnesses, it can also vary. The average for healthy adults lies between 60-100 bpm, but generally, a lower heart rate indicates a healthier and more efficient heart.

\subsubsection{Oxygen Saturation}

Blood Oxygen percentages represent the abundance of oxygen in the blood stream, which shows how well the body is distributing oxygen from the lungs to all of its cells. It is important to keep an eye on the oxygen saturation of those who suffer from chronic disease especially respiratory problems.

As they may have lower oxygen saturation levels
Table 1. Heart rate women (BPM)

\begin{tabular}{|c|c|c|c|c|}
\hline Age & poor & $\begin{array}{c}\text { Below } \\
\text { average }\end{array}$ & average & Good \\
\hline $18-25$ & $85+$ & $79-84$ & $74-78$ & $70-73$ \\
\hline $25-35$ & $83+$ & $77-82$ & $73-76$ & $59-72$ \\
\hline $36-45$ & $85+$ & $79-84$ & $74-78$ & $70-73$ \\
\hline $46-55$ & $84+$ & $78-83$ & $74-77$ & $70-73$ \\
\hline $56-65$ & $84+$ & $78-83$ & $74-77$ & $69-73$ \\
\hline $65+$ & $85+$ & $77-84$ & $73-76$ & $69-72$ \\
\hline
\end{tabular}

Table 2. Men heart rate (BPM)

\begin{tabular}{ccccc}
\hline Age & poor & $\begin{array}{c}\text { Below } \\
\text { average }\end{array}$ & average & Good \\
\hline $18-25$ & $82+$ & $74-81$ & $70-73$ & $66-69$ \\
$25-35$ & $82+$ & $75-81$ & $71-74$ & $66-70$ \\
& & & $71-75$ & $67-70$ \\
$46-45$ & $83+$ & $76-82$ & $72-76$ & $68-71$ \\
$46-55$ & $84+$ & $77-83$ & $72-75$ & $69-71$ \\
$56-65$ & $82+$ & $76-81$ & $70-73$ & $65-69$ \\
\hline $65+$ & $80+$ & $74-79$ & & \\
\hline
\end{tabular}

Table 3. Blood Oxygen saturation levels

\begin{tabular}{lll}
\hline Case & $\begin{array}{l}\text { SpO2 range } \\
(\mathbf{\%})\end{array}$ & $\begin{array}{l}\text { Healthcare } \\
\text { intervention }\end{array}$ \\
\hline Healthy adult & $95-100$ & $\begin{array}{l}\text { None needed } \\
\text { Continued }\end{array}$ \\
$\begin{array}{l}\text { Adult with } \\
\text { respiratory }\end{array}$ & $88-92$ & $\begin{array}{l}\text { assessment } \\
\text { and } \\
\text { monitoring }\end{array}$ \\
illness & & $\begin{array}{l}\text { Oxygen } \\
\text { therapy } \\
\text { needed }\end{array}$ \\
$\begin{array}{l}\text { Hypoxia } \\
\text { (oxygen } \\
\text { deprivation) }\end{array}$ & Below 88 & \\
\hline
\end{tabular}


than normal. This helps in checking the efficacy of their treatments and medicines. For Healthy adults, the average ranges from $94 \%$ to $99 \%$ and the percentage differs with respect to the age as shown in Table 3.

\subsubsection{Blood Pressure}

Blood pressure is a measure of the force that your heart uses to pump blood around your body and there is tow numbers are taken into consideration to measure the blood pressure of a patient the first systolic pressure.

This is the pressure when the heart pushes the blood out the second diastolic pressure which is the pressure when the heart rests between beats and there is a classification for the blood pressure.

- Normal: between $90 / 60 \mathrm{mmHg}$ and $120 / 80$ $\mathrm{mmHg}$.

- High blood pressure: $140 / 90 \mathrm{mmHg}$ or higher.

- Low blood pressure: 90/60 mmHg or lower.

After collecting the previous data from the patient, it passes from the biomedical kit to the Raspberry pi to be processed. Suppose any numbers appear to be out of the ordinary, according to the range mentioned above. In that case, a direct notification will be sent to the medical team, alerting them to take action.

\subsubsection{Fall Detection}

Falling can be quite dangerous for elderly people, and people with limited mobility in general. It can cause joint dislocation, bone fractures, or even bone breakage [10-13]. Fall detection systems are a simple and affordable way to immediately notify relatives or medical professionals in case of a patient's fall. They are implemented using and accelerometer and a gyroscope to calculate the angle and speed of a movement, from which we can detect if the person is falling. An additional piezo sensor is also sometimes used to detect impact.

\subsection{System Architecture}

\subsubsection{Hardware}

The hardware for this project comprises of two main parts that work together to capture the patient's vital signs, process them and subsequently save them onto the backend server to be stored in the database.

\subsubsection{Raspberry Pi:}

The core of the raspberry pi is ARM microprocessor. Our raspberry pi is based on the ARM Cortex-A53 which is a high versatility and efficient processor. For our use the Raspberry Pi we will use it as a portable computer to be connected to the biomedical kit to receive and process the data and then send if over Wi-Fi to update the database with the patient's recordings. It will also contain the system that will notify the medical team in case of any alarming vital signs.

This will be achieved through a program that periodically ( $5 \mathrm{sec}$. in the proposed scenario) checks the received data against predetermined minimum and maximum values (as listed in the tables in the previous section). if at any point the patient's vitals fall outside the normal range, a direct notification or Email will be sent to their nurse and physician without passing through the database backend server or needing to be logged into the application. The Raspberry Pi will also be used as a microcontroller to be connected to a gyroscope, accelerometer, and piezo sensor. Which in combination will be used as a fall detection sensor, because our fall detection system relies on angular velocity an acceleration, it is considered to be an appropriately accurate setup for our use.

\subsubsection{Biomedical kit (AS7030B-EVALKIT)}

This is an evaluation kit for a vital signs module by AMS called AS7030B shown in Fig.2, that contains an ECG and a PPG that can monitor heart rate, heart variability, perform cuff-less blood pressure calculation, and blood oxygen saturation. It also contains a skin temperature sensor. The raw data is then converted to readable data by a microprocessor located on the evaluation kit. Which can then be connected to any computer via USB to access the information.

\subsubsection{Software}

The software part of our system will include a database to store all of the acquired data from 
the patient, and a mobile application used by the medical team to access this data.

\subsubsection{Database}

In our case the database will be used to save any data regarding the patient or the medical team. From the complete patient history to the constantly updated patient's recordings.

A particular type of database called real-time database is designed to store information needed for real-time processing to handle workloads whose state is constantly and quickly changing. We considered hosting the DB bon Microsoft azure and Amazon web services (AWS) but opted to use Google's firebase service to design our real-time database.

Google's Firebase can be easily configured to the mobile application through android studio. For our system it will be the easier and more efficient choice since we will be designing android mobile applications to access our patient's data.

We are using two firebase storage systems, firstly is the Firestore database which more closely resembles a standard database with some optimizations for mobile applications, the other is a real-time database which can be updated instantly by any data uploaded through the application or the wearable device, such as recordings, prescriptions or general personal information about the patient.

\subsubsection{Mobile application:}

Our mobile application will include a registration page used to enter new patients and members of the medical team as shown in Fig. 3. An administrator will be in charge of assigning patients to each physician or nurse if there are several of each.

Next, each medical team member will have a page specific to them where they can access the information that pertains to them for each of their patients.

This will include three pages: the first contains patient's general information, the second contains the diagnosis and prescription, and the last will display the recordings which will be updated as soon as the medical team member opens the application.

\section{RESULTS}

As shown in Figure 3; our completed setup will consist of the raspberry pi zero $\mathrm{W}$ connected with the AS7030B-EVALKIT, as well as a gyroscope/ accelerometer and piezo sensors, which will be powered by an external rechargeable battery of around $10 \mathrm{Amp}$. that will be able to keep our system going for 56 hours.

The raspberry pi will collect the vital signs

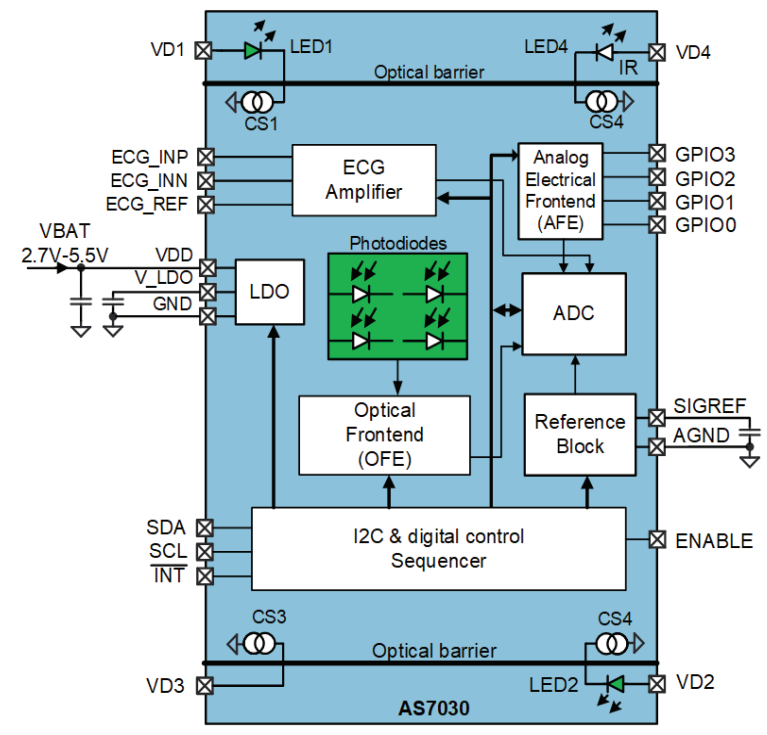

Fig 2. AS7030b block diagram 
data $\log$ from the kit then upload it to the real-time firebase database. High-level and low-level alarms for all the vital signs, as well as the fall detection system will be put in place on the raspberry pi to notify the medical team immediately in case of any unusual readings.
All the readings from our wearable device as well as the patient's history, diagnosis, and prescription will be available on the android application for the medical team in charge of the case as shown in Figure 3.

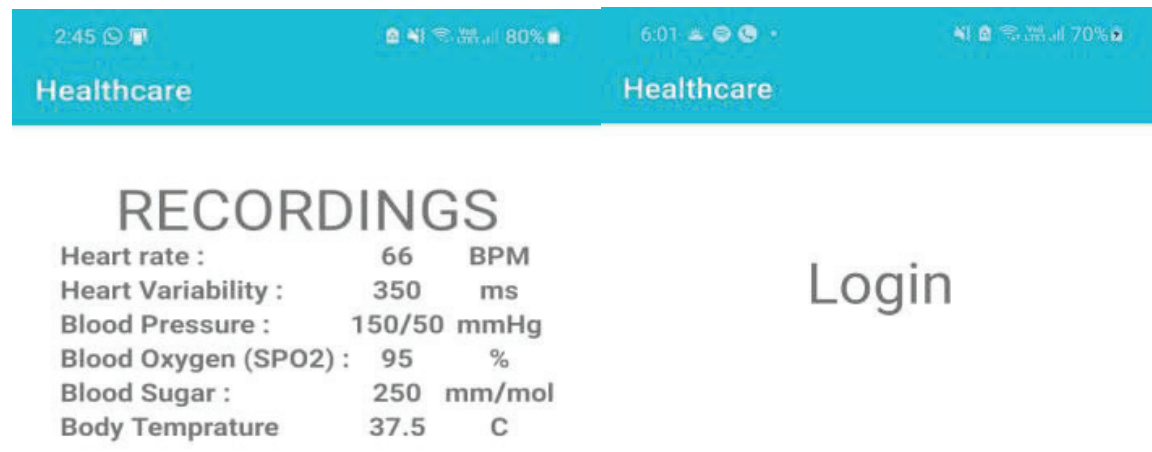

THE DIAGNOSIS

doctor@gmail.com
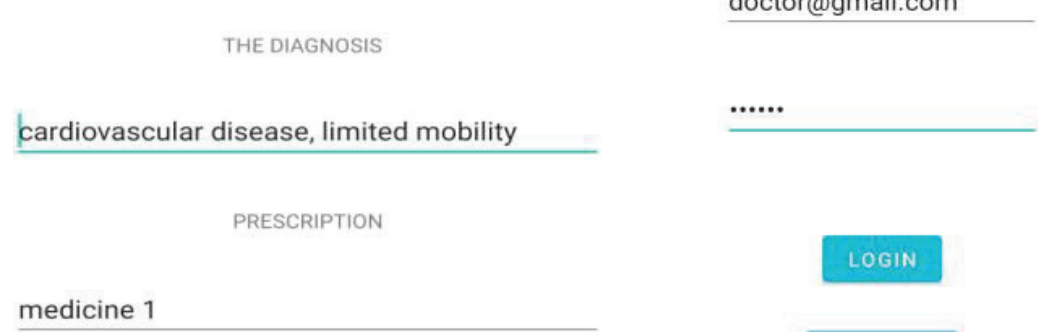

\section{LOGIN}

REGISTER

\section{UPDATE}

III

○
$<$

Fig 3. Mobile application user interface

\section{CONCLUSION}

In brief, our goal is to design, simulate, and implement a connected IoT-HealthCare system that is easily monitored and controlled through a mobile application, and an integrated wearable device that detects and records the vital signs of the patient, to help keep an eye on the patient without the need for constant physical presence. This paper aims to design, simulate, and implement a connected IoT-Health Care system that assists patients' need in terms of caring and monitoring. A proof of concept implementation was demonstrated using raspberry Pi, Google Firebase DB, and android OS. Initial results show the promise of such a system to help patients. Our future work is having this contribution applicable to real case patients with specific scenarios

\section{ACKNOWLEDGMENT}

I would like to express my special thanks of gratitude to the Reviewers, who gave their valuable comments that were a reason in enhancing this work. Moreover, I would show my appreciation to the Editor PAS for his vital countless effort.

\section{CONFLICT OF INTEREST}

The authors declare no conflict of interest. 


\section{REFERENCES}

1. Darshan, Anandakumar, "A comprehensive review on usage of internet of things (IoT) in healthcare system", (2015).

2. M. A. Azzawi., R. Hassan., K. A. Bakar., "A review on internet of things (IoT) in healthcare", Selangor, Malaysia (November 2016).

3. Z. Alansari., S. Soomro., M. R. Belgaum, S. Shamshirband. "The rise of internet of things (IoT) in big healthcare data: review and open research issues", Singapore, Malaysia (2017).

4. I. Chiuchisan., H. N. Costin., and O. Geman. "Adopting the Internet of Things Technologies in Health Care Systems", (2014).

5. A. A. Albesher. "IoT in Health-care: Recent Advances in the Development of Smart CyberPhysical Ubiquitous Environments", (2019).

6. P. Gupta., D. Agrawal, J. Chhabra., P. K. Dhir. "IoT based smart healthcare kit", (2016).

7. M. P. R. S. Kiran, P. Rajalakshmi, K. Bharadwaj and A. Acharyya. "Adaptive Rule Engine Based IoT Enabled Remote Health Care Data Acquisition and Smart Transmission System", (2014).

8. K. Guk, G. Han, J. Lim, K. Jeong, T. Kang, E. K. Lim, J. Jung, "Evolution of Wearable Devices with Real-Time Disease Monitoring for Personalized Healthcare", (29 May 2019).

9. R.A. Khan., A. K. Pathan. "The state-of-the-art wireless body area sensor networks", (2018).

10. L. Aminzadeh., K. AlOtoon., A. Al Dmour., K. Elleithy. "A panoramic study of fall detection technologies", (May 2016).

11. X. Wang. J. Ellul., G. Azzopardi. "Elderly fall detection systems: A literature survey", (2020).

12. J. Miranda.,J. Cabral., S. R.Wagner., C.F.Pedersen., B.Ravelo., M. Memon., and M.Mathiesen. "An Open Platform for Seamless Sensor Support in Healthcare for the Internet of Things", (2016)

13. A. Sangwan., P. P. Bhattacharya. "Wireless body sensor networks: a review", (2015). 
